\title{
SDR Verification of Hierarchical Decision Aided 2-Source BPSK H-MAC CSE with Feed-Back Gradient Solver for WPNC Networks
}

\author{
Petr HRON, Jozef LUKAC, Jan SYKORA \\ Dept. of Radio Radioelectronics, Czech Technical University in Prague, Technicka 2, 16627 Praha, Czech Republic \\ hronpetr@fel.cvut.cz, lukacjo1@fel.cvut.cz, jan.sykora@fel.cvut.cz
}

Submitted December 29, 2019 / Accepted June 1, 2020

\begin{abstract}
This paper considers a channel state estimation (CSE) problem in a parametrized Hierarchical MAC $(\mathrm{H}$ MAC) stage in Wireless Physical Layer Network Coding (WPNC) networks with Hierarchical Decode and Forward $(H D F)$ relay strategy. The primary purpose is to present the results of a non-pilot based phase estimator performance evaluation. In particular, the performance comparison of a MATLAB simulation and an over the air transmission using USRP N210 transceivers in terms of mean square error (MSE) and bit error rate (BER). Also, we analyze the properties of the Cramer Rao Lower Bound (CRLB) w.r.t. different channel parametrizations.
\end{abstract}

\section{Keywords}

Software Defined Radio (SDR), H-MAC CSE, WPNC, phase, estimator

\section{Introduction}

Wireless Physical Layer Network Coding (WPNC) is a PHY layer concept for communication in dense radio networks with highly interacting signals. We have reached a relatively solid understanding of some fundamental limits, the system design and performance analysis in basic topologies and scenarios [1-5]. The knowledge includes achievable and converse rates for specific strategies and scenarios (e.g., Compute \& Forward, Noisy Network Coding, Hierarchical Decode and Forward (HDF)), design of Network Coded Modulation (NCM) and related hierarchical demodulation and decoding strategies, including their performance analysis. Most of the results assume relatively idealized assumptions related to relative channel parametrization, which dramatically affects the resulting hierarchical constellation in a nonlinear way. Thus it is desirable to track the channel parameters even while payload data is transmitted.
This paper builds on the results of [6], and [7] and concentrates on a practical evaluation of the proposed feed-back gradient solver of the hierarchical phase estimator.

In WPNC, the word "hierarchical" is used to emphasize that receivers observe several interacting signals at the same time, at the same carrier frequency. It is one of the leading principles of WPNC - the exploiting of the information carried by interference. Following this paradigm, signals from different sources cannot be observed independently. The paper [6] derives a phase estimator individual signals based only on their non-orthogonal superposition and $\mathrm{H}$-data symbols. In this work we analyze this estimator in detail. We compare the over-the-air performance to a simulation in terms of the mean square error (MSE) and the bit-error-rate (BER) for an uncoded transmission. Besides, we analyze and present the dependence of the Cramer-Rao Lower Bound (CRLB) on the channel state.

The rest of this paper is organized as follows. In Sec. 2, we introduce the system and channel model considered in the rest of the paper. Section 3 summarizes the results of [6], mainly the phase estimator and gradient descent solver. In Sec. 4 , we present some properties of the constant phase estimator CRLB. Sections 5 and 6 describe the considered scenarios and the experimental setup based on USRP transceivers. Section 7 presents and discusses the results of the experiment and simulation. Finally in Sec. 8 we conclude the paper.

\section{System Model}

In this work, we assume a three-node network with two source nodes (SA, SB) and one relay R, see Fig. 1. Further, we assume a perfect symbol-timing synchronization among all three nodes. It is an idealized situation that is though to ensure in practice. We have chosen it to simplify the problem. A system model with asynchronous channels and the estimation of the channel delays is considered in e.g., [8] and [9]. 


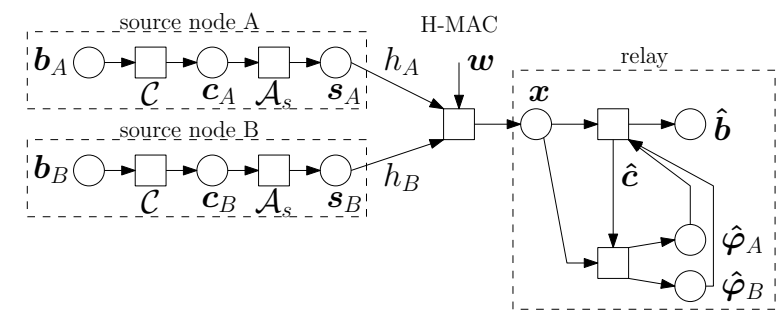

Fig. 1. System model as given in [6].

The network operates at the H-MAC stage, where the interaction between both source signals is allowed, and the relay's target is a many-to-one function (Hierarchical Network Code (HNC) map) of the individual source messages $b=\chi\left(b_{A}, b_{B}\right)$. The processing at each source node is, in general, as follows. The source message is encoded by a common codebook $C, \mathbf{c}_{A}=C\left(b_{A}\right)$ and symbol-wise mapped on the constellation points $s_{A, n}=s_{A}\left(c_{A, n}\right)$ using a one-dimensional alphabet $\mathcal{A}_{s}$ of size $M_{s}$. Similarly for SB. The target H-code symbols are denoted as $\mathbf{c}=C(b)$. We assume an isomorphic layered NCM with a minimal HNC map. The isomorphic layered property implies that $c_{n}=\chi_{c}\left(c_{A, n}, c_{B, n}\right)$ and the minimality ensures that the elements of $\left\{c_{n}, c_{A, n}, c_{B, n}\right\}$ are coupled in such a way that there are only two degrees of freedom, i.e. given arbitrary two, the third is unambiguously determined.

We assume a memoryless 2-source H-MAC AWGN channel model given as

$x_{n}=u\left(c_{A, n}, c_{B, n}\right)+w_{n}=h_{A, n} s_{A}\left(c_{A, n}\right)+h_{B, n} s_{B}\left(c_{B, n}\right)+w_{n}$

where the fading coefficients are decomposed into magnitude and phase $h_{A, n}=\mathrm{e}^{\mathrm{j} \varphi_{A, n}}, h_{B}=\eta \mathrm{e}^{\mathrm{j} \varphi_{B, n}}, \eta \in \mathbb{R}^{+}$and $w_{n}$ is AWGN with $\sigma_{w}^{2}$ variance per dimension. The observed frame is of length $N$. The SNR will be related w.r.t. SA and denoted as $\gamma_{x}=\mathrm{E}\left[\left|s_{A}\right|^{2}\right] / \sigma_{w}^{2}$.

In this paper we consider a special case of a $B P S K$ alphabet $\mathcal{A}_{S}=\{ \pm 1\}$ and binary coded symbols $c_{A}, c_{B} \in\{0,1\}$ on both source nodes. The natural $\mathrm{H}$-constellation mapper $s(c)=2 c-1$ is used. In this special case it follows, that the only minimal HNC map is accomplished by the XOR function $c=c_{A} \oplus c_{B}, c \in\{0,1\}$.

\section{ML Phase Estimator}

In [6] the hierarchical symbol conditioned channel phases likelihood is derived as

$$
\begin{gathered}
\mathrm{p}\left(x_{n} \mid \check{\varphi}_{A, n}, \check{\varphi}_{B, n}, c_{n}\right) \propto \exp \left(-\frac{2}{\sigma_{w}^{2}} \eta s\left(c_{n}\right) \cos \left(\check{\varphi}_{A, n}-\check{\varphi}_{B, n}\right)\right) \\
2 \cosh \left(\frac{2}{\sigma_{w}^{2}}\left(\mathfrak{R}\left[x_{n} \mathrm{e}^{-\mathrm{j} \check{\varphi}_{A, n}}\right]+\eta s\left(c_{n}\right) \mathfrak{R}\left[x_{n} \mathrm{e}^{-\mathrm{j} \check{\varphi}_{B, n}}\right]\right)\right)
\end{gathered}
$$

where $s(c)=s_{A} s_{B}^{*}=1-2 c$. This expression is obtained after a marginalization of $\mathrm{p}\left(x \mid \check{\varphi}_{A}, \breve{\varphi}_{B}, s_{A}, s_{B}\right)$ over the hierarchical dispersion i.e. the uncertainty of $s_{A}\left(c_{A}\right), s_{B}\left(c_{B}\right)$ given $c=\chi\left(c_{A}, c_{B}\right)$. Assuming $\varphi_{A}, \varphi_{B}$ constant over the whole frame of $N$ symbols, the ML estimator is given as

$$
\left[\hat{\varphi}_{A}, \hat{\varphi}_{B}\right]=\arg \max _{\left[\check{\varphi}_{A}, \check{\varphi}_{B}\right]} \sum_{n=1}^{N} \rho\left(\check{\varphi}_{A}, \check{\varphi}_{B}, c_{n}, x_{n}\right)
$$

where $\rho\left(\check{\varphi}_{A}, \check{\varphi}_{B}, c_{n}, x_{n}\right)$ is the log-likelihood which we obtain as

$$
\begin{gathered}
\rho\left(\check{\varphi}_{A}, \check{\varphi}_{B}, c_{n}, x_{n}\right)=-\frac{2}{\sigma_{w}^{2}} \eta s\left(c_{n}\right) \cos \left(\check{\varphi}_{A}-\check{\varphi}_{B}\right)+ \\
\ln \cosh \left(\frac{2}{\sigma_{w}^{2}}\left(\mathfrak{R}\left[x_{n} \mathrm{e}^{-\mathrm{j} \check{\varphi}_{A}}\right]+\eta s\left(c_{n}\right) \Re\left[x_{n} \mathrm{e}^{-\mathrm{j} \check{\varphi}_{B}}\right]\right)\right) .
\end{gathered}
$$

For finding the argument of the maximum we use an iterative gradient descent algorithm given as

$$
\hat{\varphi}(i+1)=\hat{\varphi}(i)+\left.\mathbf{K} \nabla_{\breve{\varphi}}\left(\sum_{n=1}^{N} \rho\left(\check{\varphi}_{A}, \check{\varphi}_{B}, c_{n}, x_{n}\right)\right)\right|_{\check{\varphi}=\hat{\varphi}(i)} .
$$

Next we consider a more practical scenario, where the phase $\varphi_{n}=\left[\varphi_{A, n}, \varphi_{B, n}\right]$ varies over the frame. For this case, we modify the full-frame gradient descent search and iterate over $\hat{\varphi}_{n}$, in each step applying a correction based on the gradient of a local neighborhood given by a window of length $W$.

$$
\hat{\boldsymbol{\varphi}}_{i+1}=\hat{\boldsymbol{\varphi}}_{i}+\left.\mathbf{K} \nabla_{\check{\varphi}}\left(\sum_{n=i-W-1}^{i-1} \rho\left(\varphi_{A, n}, \varphi_{B, n}, c_{n}, x_{n}\right)\right)\right|_{\check{\varphi}=\hat{\varphi}_{i}} .
$$

The window length should reflect the amount of change of the phase between two consecutive samples. Our implementation of this approach assumes that the initial phase is known to the estimator i.e. $\hat{\varphi}_{1}=\varphi_{1}$.

\section{Cramer-Rao Lower Bound Properties}

The CRLB of an ML constant phase estimator was evaluated in [7] for a specific pair of phases and a relative channel attenuation. We present the dependence of the CRLB on SNR, $\eta$ and the phase difference.

In the Figs. 2, 3, 4 and 5, we see a numerical evaluation of the constant phase estimator CRLB for different SNR and relative channel attenuations $\eta$. Further, we present some properties of the CRLB. 
1. The CRLB depends on the phase difference $\Delta \varphi=\varphi_{A}-\varphi_{B}$ only.

The metric $\rho$ is a logarithm of the likelihood, thus for the evaluation of the Fisher information matrix (7) can be used.

$$
\mathbf{J}=\mathrm{E}\left[\left(\begin{array}{cc}
\frac{\partial \rho}{\partial \varphi_{A}} \frac{\partial \rho}{\partial \varphi_{A}}, & \frac{\partial \rho}{\partial \varphi_{A}} \frac{\partial \rho}{\partial \varphi_{B}} \\
\frac{\partial \rho}{\partial \varphi_{B}} \frac{\partial \rho}{\partial \varphi_{A}}, & \frac{\partial \rho}{\partial \varphi_{B}} \frac{\partial \rho}{\partial \varphi_{B}}
\end{array}\right)\right] .
$$

In this section we relax the notation and use $\varphi_{A}, \varphi_{B}$ instead of $\check{\varphi_{A}}, \breve{\varphi_{B}}$. The components of the gradient of $\rho$, $\mu_{A}:=\partial \rho / \partial \varphi_{A}$ and $\mu_{B}:=\partial \rho / \partial \varphi_{B}$ are evaluated in [6] together with their low SNR and high SNR approximations. We consider the metric (4). For notation clarity we drop the explicit index $n$. The gradient components of the metric (4) are (8), (9).

$$
\begin{gathered}
\mu_{A}=\frac{2}{\sigma_{w}^{2}} \eta s(c) \sin \left(\varphi_{A}-\varphi_{B}\right)+\frac{2}{\sigma_{w}^{2}} \mathfrak{J}\left[x \mathrm{e}^{-\mathrm{j} \varphi_{A}}\right] \\
\tanh \left(\frac{2}{\sigma_{w}^{2}} \mathfrak{R}\left[x \mathrm{e}^{-\mathrm{j} \varphi_{A}}+\eta s(c) x \mathrm{e}^{-\mathrm{j} \varphi_{B}}\right]\right), \\
\mu_{B}=-\frac{2}{\sigma_{w}^{2}} \eta s(c) \sin \left(\varphi_{A}-\varphi_{B}\right)+\frac{2}{\sigma_{w}^{2}} s(c) \eta \mathfrak{J}\left[x \mathrm{e}^{-\mathrm{j} \varphi_{B}}\right] \\
\tanh \left(\frac{2}{\sigma_{w}^{2}} \mathfrak{R}\left[x \mathrm{e}^{-\mathrm{j} \varphi_{A}}+\eta s(c) x \mathrm{e}^{-\mathrm{j} \varphi_{B}}\right]\right) .
\end{gathered}
$$

For the computation of the Fisher matrix (7), we evaluate the expected value over all contained random variables, i.e. over $x$ and $s(c)$. For the purpose of the computation we substitute the channel model $x:=\exp \left(\mathrm{j} \varphi_{A}\right) s_{A}+\eta \exp \left(\mathrm{j} \varphi_{B}\right) s_{B}+w$ and $s(c):=s_{A} s_{B}$ (we drop the conjugation because $s_{B}$ is real valued) into the gradient components (8), (9). Further we use $\Delta \varphi:=\varphi_{A}-\varphi_{B}$. After some manipulation we get (10), (11).

$$
\begin{aligned}
\mu_{A} & =\frac{2}{\sigma_{w}^{2}} \eta s_{A} s_{B} \sin (\Delta \varphi)+\frac{2}{\sigma_{w}^{2}} \mathfrak{J}\left[s_{A}+\eta s_{B} \mathrm{e}^{-\mathrm{j} \Delta \varphi}\right. \\
& \left.+w \mathrm{e}^{-\mathrm{j} \varphi_{A}}\right] \tanh \left(\frac { 2 } { \sigma _ { w } ^ { 2 } } \mathfrak { R } \left[s_{A}\left(1+\eta^{2}\right)\right.\right. \\
& \left.\left.+s_{B} \eta\left(\mathrm{e}^{-\mathrm{j} \Delta \varphi}+\mathrm{e}^{\mathrm{j} \Delta \varphi}\right)+w \mathrm{e}^{-\mathrm{j} \varphi_{A}}\left(1+\eta s_{A} s_{B} \mathrm{e}^{\mathrm{j} \Delta \varphi}\right)\right]\right),
\end{aligned}
$$

$$
\begin{aligned}
\mu_{B} & =-\frac{2}{\sigma_{w}^{2}} \eta s_{A} s_{B} \sin (\Delta \varphi)+\frac{2}{\sigma_{w}^{2}} \eta \mathfrak{I}\left[s_{B} \mathrm{e}^{\mathrm{j} \Delta \varphi}+\eta s_{A}\right. \\
& \left.+w \mathrm{e}^{-\mathrm{j} \varphi_{A}} s_{A} s_{B} \mathrm{e}^{\mathrm{j} \Delta \varphi}\right] \tanh \left(\frac { 2 } { \sigma _ { w } ^ { 2 } } \mathfrak { R } \left[s_{A}\left(1+\eta^{2}\right)\right.\right. \\
& \left.\left.+s_{B} \eta\left(\mathrm{e}^{-\mathrm{j} \Delta \varphi}+\mathrm{e}^{\mathrm{j} \Delta \varphi}\right)+w \mathrm{e}^{-\mathrm{j} \varphi_{A}}\left(1+\eta s_{A} s_{B} \mathrm{e}^{\mathrm{j} \Delta \varphi}\right)\right]\right) .
\end{aligned}
$$

Because a complex white Gaussian random variable $w$ is rotationally invariant, the distribution of its rotated version $w$, i.e. $w \exp \left(-\mathrm{j} \varphi_{A}\right)$, is identical to distribution of $w$ for an arbitrary fixed $\varphi_{A}$. In the computation of (7), we can assign $w^{\prime}:=w \exp \left(-\mathrm{j} \varphi_{A}\right)$ and evaluate the expectation w.r.t. $w^{\prime}$ instead of $w$. This means that the Fisher matrix $J=J\left(\Delta \varphi, \varphi_{A}\right)$ is equal to $J(\Delta \varphi, 0)$ for every $\varphi_{A}$, i.e. it does not depend on $\varphi_{A}$.

2. The CRLB is a periodic function of $\Delta \varphi$ with period $\pi$. Clearly, it is periodic with the period $2 \pi$. We just need to show that $J(\Delta \varphi)=J(\Delta \varphi+\pi)$. When substituting $\Delta \varphi:=\Delta \varphi+\pi$ in the gradient components (10), (11), we get $\exp [\mathrm{j}( \pm \Delta \varphi \pm \pi)]=-\exp [ \pm \mathrm{j} \Delta \varphi]$. Then we substitute $s_{B}^{\prime}:=-s_{B}$. The distribution of $s_{B}^{\prime}$ is identical to the distribution of $s_{B}$, hence $J(\Delta \varphi)=J(\Delta \varphi+\pi)$. The periodicity can be seen in Fig. 2.

3. The CRLB of both $\varphi_{A}$ and $\varphi_{B}$ for $\Delta \varphi=0$ and $\eta=1$ is infinite.

When $\Delta \varphi=0$ (i.e. $\varphi_{A}=\varphi_{B}$ ) and $\eta=1$, the gradient component $\mu_{B}$ (11) is a multiple of $\mu_{A}$ (10): $\mu_{B}=\mu_{A} s_{A} s_{B}$. Then it can be shown (by Gaussian elimination), that $J$ is singular (12).

$$
\begin{aligned}
J & =\mathrm{E}\left[\mu_{A}^{2}\left(\begin{array}{cc}
1 & s_{A} s_{B} \\
s_{A} s_{B} & 1
\end{array}\right)\right] \\
& =\mathrm{E}\left[\frac{\mu_{A}^{2}}{s_{A} s_{B}}\left(\begin{array}{cc}
1 & 1 \\
0 & s_{A} s_{B}
\end{array}\right)\left(\begin{array}{cc}
s_{A} s_{B} & -1 \\
0 & 1
\end{array}\right)\right. \\
& \left.\left(\begin{array}{cc}
1 & s_{A} s_{B} \\
s_{A} s_{B} & 1
\end{array}\right)\right] \\
& =\mathrm{E}\left[\frac{\mu_{A}^{2}}{s_{A} s_{B}}\left(\begin{array}{cc}
1 & 1 \\
0 & s_{A} s_{B}
\end{array}\right)\left(\begin{array}{cc}
0 & 0 \\
s_{A} s_{B} & 1
\end{array}\right)\right] .
\end{aligned}
$$

Its inverse does not exist, resp. in the limit, the elements of $J$ grow (in absolute value) to infinity (non-diagonal elements can be negative).

From Figs. 2, 3, 4 and 5 we can further see:

1. the CRLB seems to be an even function of $\Delta \varphi$,

2. there are two main areas of $\Delta \varphi$ :

(a) $|\Delta \varphi-k \pi|$ is greater than cca. $0.15 \pi[\mathrm{rad}], k \in \mathbb{Z}$.

(b) $|\Delta \varphi-k \pi|$ is lower than cca. $0.15 \pi[\mathrm{rad}], k \in \mathbb{Z}$.

For case (a), the CRLB for channel $A\left(\operatorname{crlb}\left[\varphi_{A}\right]\right)$ does practically not depend on $\eta$, the CRLB for channel $B$ $\left(\mathrm{crlb}\left[\varphi_{B}\right]\right)$ decreases with increasing $\eta$. For case (b), the CRLB for channel $A$ increases with increasing $\eta$, the CRLB for channel $B$ stays larger than the CRLB for channel $A$ for corresponding $\eta$.

3. we can also notice that the peaks of the CRLB fade for increasing SNR. 


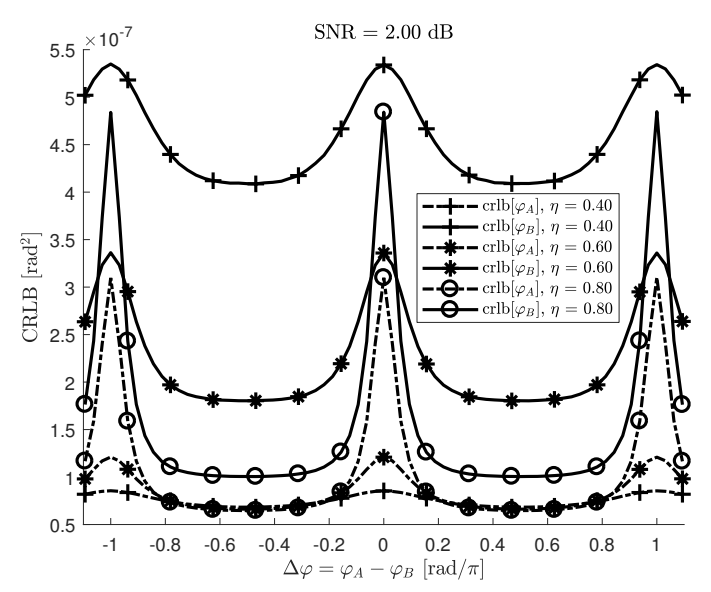

Fig. 2. CRLB of the constant phase estimator for $\mathrm{SNR}=2 \mathrm{~dB}$ and relative channel gains $\eta$. Evaluated for $\varphi_{A}=\pi / 4 \mathrm{rad}$.

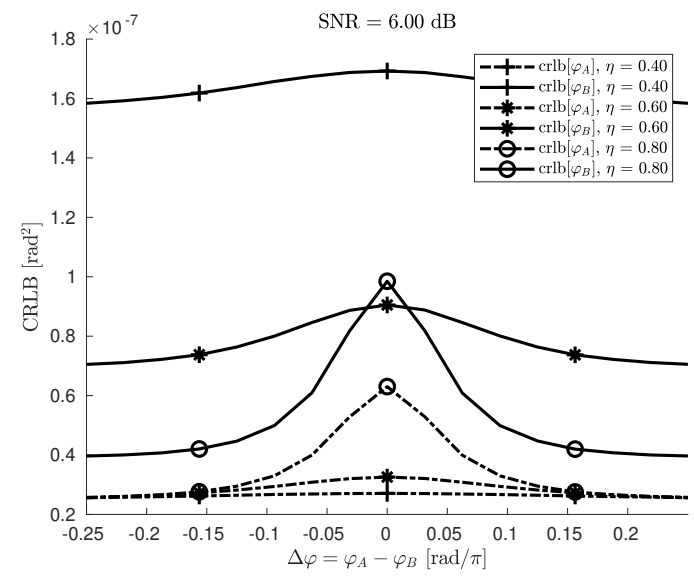

Fig. 4. CRLB of the constant phase estimator for $\mathrm{SNR}=6 \mathrm{~dB}$ and relative channel gains $\eta$. Evaluated for $\varphi_{A}=\pi / 4 \mathrm{rad}$.

\section{Considered Scenarios}

The primary purpose of this article is to evaluate the performance of the proposed estimator using an over-the-air transmission and compare it with simulation results. For this purpose, we considered multiple scenarios and assessed the MSE of the phase estimates and BER of the hierarchical message $\mathbf{c}$ for different values of SNR. Because the final goal of the relay processing is an estimate of the hierarchical c, the classical MSE of the form $\mathrm{E}\left[(\boldsymbol{\varphi}-\hat{\varphi})^{2}\right]$ is not suitable because the many-to-one $\chi$ function introduces a many to one relationship between channel phase and observation. In particular, it holds that [7]:

$$
u(\varphi, c)=u\left(\varphi+\left[\left(2 k_{1}+1\right) \pi,\left(2 k_{2}+1\right) \pi\right], c\right), k_{1}, k_{2} \in \mathbb{Z} .
$$

To account for this, we evaluate the MSE as

$$
\min _{k_{1}, k_{2}} \mathrm{E}\left[\left(\boldsymbol{\varphi}-\hat{\boldsymbol{\varphi}}+\left[\left(2 k_{1}+1\right) \pi,\left(2 k_{2}+1\right) \pi\right]\right)^{2}\right] \text {. }
$$

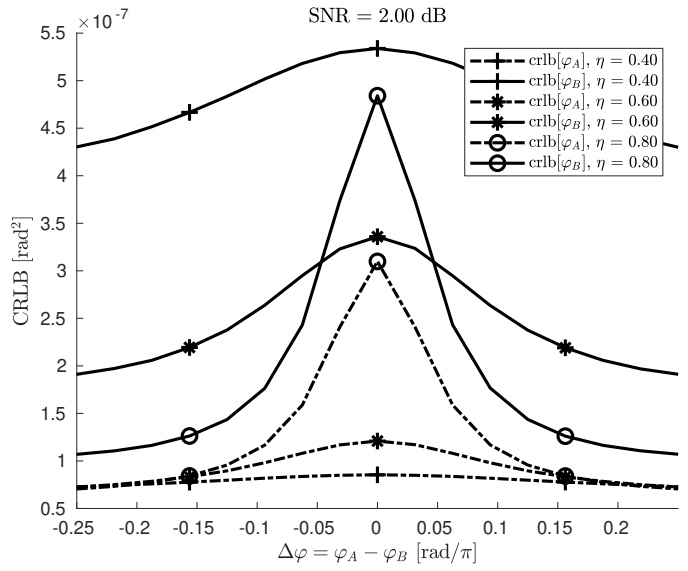

Fig. 3. CRLB of the constant phase estimator for $\mathrm{SNR}=2 \mathrm{~dB}$ and relative channel gains $\eta$. Evaluated for $\varphi_{A}=\pi / 4 \mathrm{rad}$.

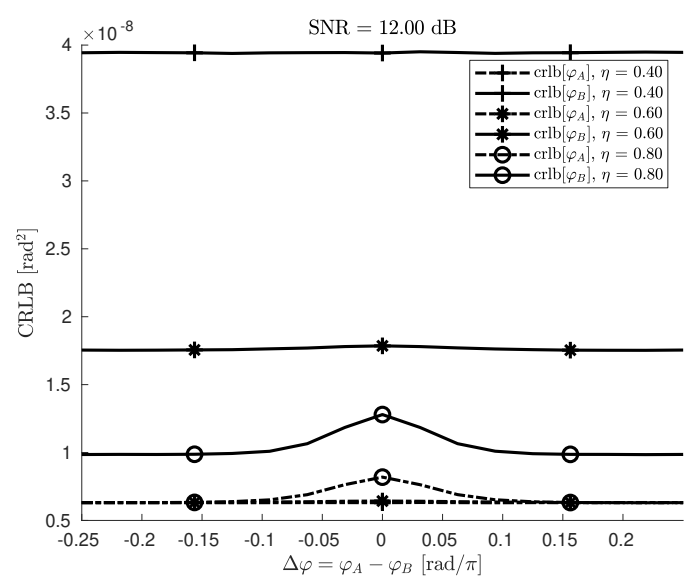

Fig. 5. CRLB of the constant phase estimator for $\mathrm{SNR}=12 \mathrm{~dB}$ and relative channel gains $\eta$. Evaluated for $\varphi_{A}=\pi / 4 \mathrm{rad}$.

\subsection{True Hierarchical Data-Aided Estimation}

In the first setting, we let the estimator know the hierarchical target message c. This scenario should produce the best result and set a performance limit, but can not be practically implemented.

\subsection{Hierarchical Data Estimated using Hard Decision}

This case resembles a practical implementation, where the hierarchical target message is not available at the relay. The phase estimator uses only an estimate $\hat{\mathbf{c}}$. In our case, we used a hard decision

$$
\hat{c}_{n}= \begin{cases}1 & \text { when } \log \left(\frac{\mathrm{p}\left(x_{n} \mid c_{n}=0\right)}{\mathrm{p}\left(x_{n} \mid c_{n}=1\right)}\right) \geq 0 \\ 0 & \text { when } \log \left(\frac{\mathrm{p}\left(x_{n} \mid c_{n}=0\right)}{\mathrm{p}\left(x_{n} \mid c_{n}=1\right)}\right)<0 .\end{cases}
$$


In our setting the values of $c_{n}$ are equiprobable, thus we have

$$
\begin{aligned}
\mathrm{p}\left(x_{n} \mid \check{c}\right) \propto \sum_{\left(c_{A}, c_{B}\right): \chi\left(c_{A}, c_{B}\right)=\check{c}} \mathrm{p}\left(x_{n} \mid c_{A}, c_{B}\right) \mathrm{p}\left(c_{A}, c_{B}\right) \\
\propto \sum_{\left(c_{A}, c_{B}\right): \chi\left(c_{A}, c_{B}\right)=\check{c}} \exp \left(\left|x_{n}-u_{n}\left(c_{A}, c_{B}\right)\right|^{2}\right) .
\end{aligned}
$$

For details see [1]

\section{Over-the-Air Implementation}

For evaluation of the estimator performance in a realworld setting, we used three software-defined radios. Each for one node SA, SB, and R. The particular type used in our experiments was the Ettus Research N210 transceiver. The experimental setting was made up of three stationary antennas placed in a time-invariant office environment, according to Fig. 6. For carrier frequency and symbol timing synchronization, we employed the OctoClock clock distribution module. The parameter setup used for our transmissions is summarized in Tab. 1. Because the estimator is unbiased, the phase estimate MSE is equal to the estimate variance. In a real scenario, the variance would have to be estimated using temporal averaging over an interval of constant channel phase. In our experimental setup, the transceivers were synchronized, and the environment assumed invariant such that we were able to work directly with the true channel phase. In the initial phase, we used one orthogonal pilot for each link SA-R, SB-R, to estimate the constant channel coefficients $h_{A}, h_{B}$. Then we modeled the variable phase $\varphi_{A, n}^{\prime}, \varphi_{B, n}^{\prime}$ as a random walk process and embedded it artificially in the generated signals such that

$$
\varphi_{n}=\left[\angle h_{A}+\varphi_{A, n}^{\prime}, \angle h_{B}+\varphi_{B, n}^{\prime}\right]
$$

In Fig. 7, we see a typical realization of the generated phase. For the initial phase channel parameter estimation, we used a $N_{p}=5 \times 10^{5}$ symbols long random, noise-like pilot sequence and employed a standard ML estimator

$$
\begin{aligned}
\angle h_{A} & =\angle\langle\mathbf{x}, \mathbf{p}\rangle, \\
\left|h_{A}\right| & =\frac{|\langle\mathbf{x}, \mathbf{p}\rangle|}{\|\mathbf{p}\|}, \\
\hat{\sigma}_{w}^{2} & =\operatorname{var}\left(\mathbf{x}-h_{A} \mathbf{p}\right)
\end{aligned}
$$

where $\mathbf{p}$ is the pilot and $\mathbf{x}$ the received signal. Similarly, for $h_{B}$. To fit our channel model (1), where source SA is assumed to have unit channel gain, we applied a suitable normalization. Desired values of $\eta$ and SNR of the normalized channel were achieved by the appropriately setting of the transmit gains of both sources SA and SB.

\section{$S A$}

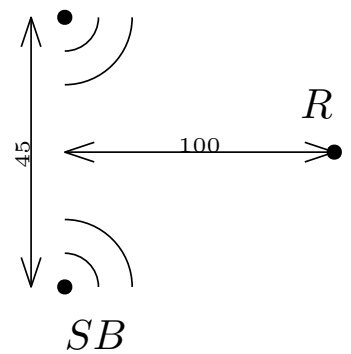

Fig. 6. Antenna arrangement.

\begin{tabular}{|c|c|}
\hline Parameter & Value \\
\hline carrier frequency & $2.4 \mathrm{GHz}$ \\
\hline sampling frequency & $10 \mathrm{MHz}$ \\
\hline modulation pulse & $\mathrm{RRC}$ \\
\hline roll-off factor & 0.35 \\
\hline samples per symbol & 8 \\
\hline
\end{tabular}

Tab. 1. Settings used for over-the-air transmissions.

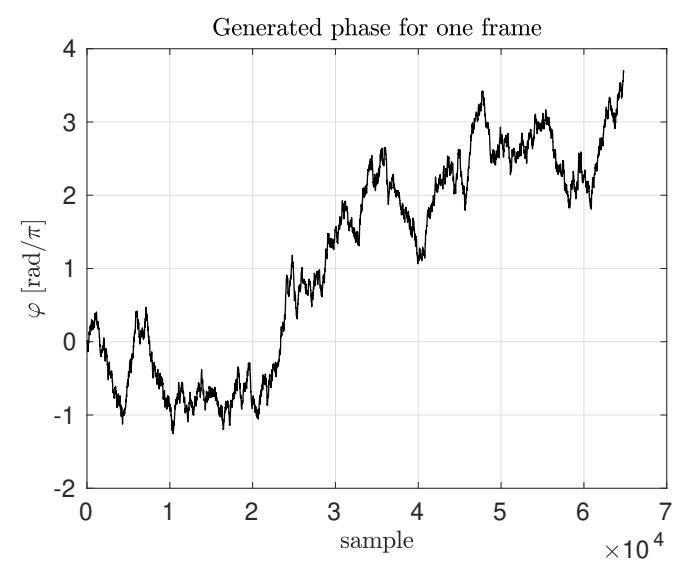

Fig. 7. Example of generated phase time dependence.

\section{Results}

In this section, we present the results of simulations using the channel according to (1) and real over-the-air transmissions conducted as described in Sec. 6. In Fig. 8, we present the evaluation of the MSE as elaborated in Sec. 5 for different values of SNR. For clarity, we plotted only the phase $\varphi_{A}$. We see that the simulation matches well with the experiment, however in contrast with the results of Sec. 4, we get worse MSE values for higher values of $\eta$. This behavior can be explained based on the results of [7]. There was shown that the estimator metric exhibits two maxima, one for the expected channel phases and a second for their flipped version. For small values of $\eta$, the maximum corresponding to the true channel phase has a larger value than the second maximum. As, however, $\eta$ approaches to 1 , both maxima become equally valued, and thus the estimator may converge to the flipped phase vector. To disregard this behavior, we define a modified MSE (denoted by a star) in such a way, that it is immune to a possible phase flip. In Fig. 9, we run the same simulations employing $\mathrm{MSE}^{*}$ and see that the results agree with Sec. 4. From [7], we also know, that for $\eta$ approaching 1, 
the observations for the true phase and the flipped version get similar and for $\eta=1$, we get

$$
u\left(c, \varphi_{A}, \varphi_{B}\right)=u\left(c, \varphi_{B}, \varphi_{A}\right) .
$$

In the BER evaluation in Fig. 12 we see, that those two effects act counter each other, such that the resulting performance in terms of BER follows the tendency that for higher $\eta$ we get better performance. Again, we see that the simulation and real-world experiment match each other well. In Fig. 10 we show the MSE* for both phases and $\eta=0.5$. We see that in accordance with Sec. 4, we get better performance for the stronger signal in both the simulation and experiment. We also evaluated the performance difference between the two cases. One where we supplied correct hierarchical data to the estimator and the second where we used hard-decision estimates only. The results are presented in Fig. 11, which shows that the aided case performs better, as anticipated. For high SNR, however, both curves merge as the hard-decision error probability drops.

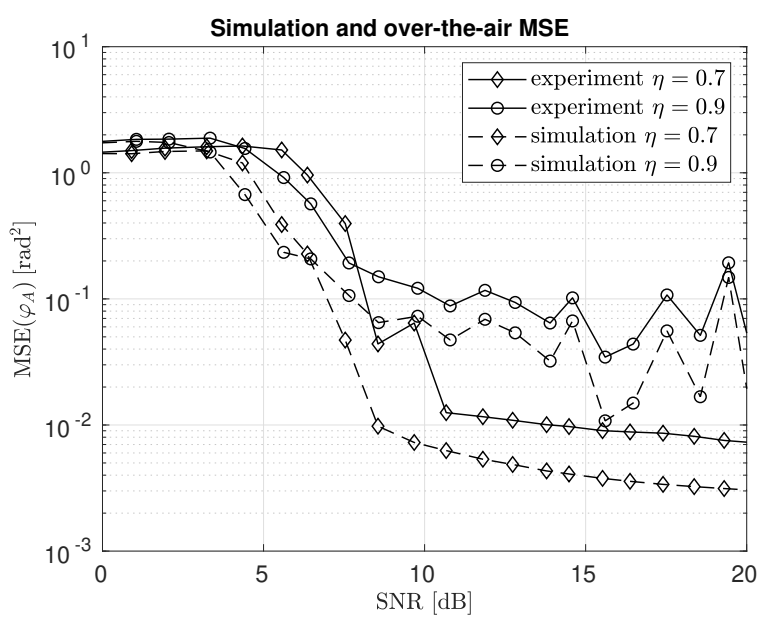

Fig. 8. Comparison of simulation and over-the-air experiment in terms of MSE of $\varphi_{A}$ for different values of $\eta \in\{0.7,0.9\}$.

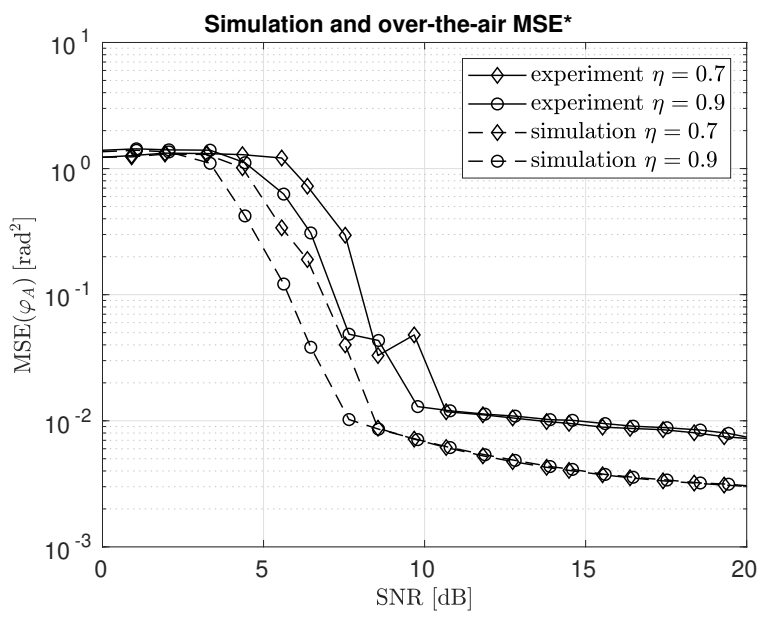

Fig. 9. Comparison of simulation and over-the-air experiment in terms of $\mathrm{MSE}^{*}$ of $\varphi_{A}$ for different values of $\eta \in\{0.7,0.9\}$. The modified MSE* resolves incorrect flipping of $\varphi_{A}$ and $\varphi_{B}$.

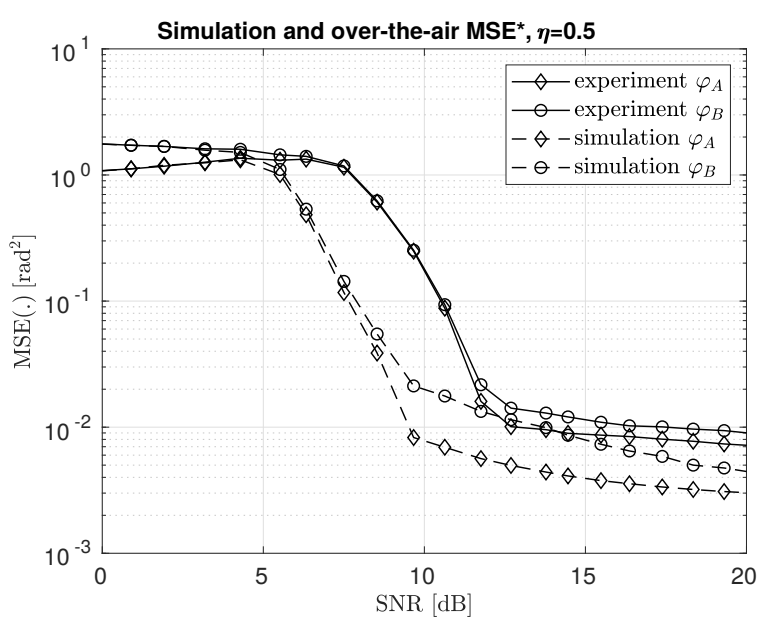

Fig. 10. Comparison of simulation and over-the-air experiment in terms of MSE* of both $\varphi_{A}, \varphi_{B}$ for $\eta=0.5$.

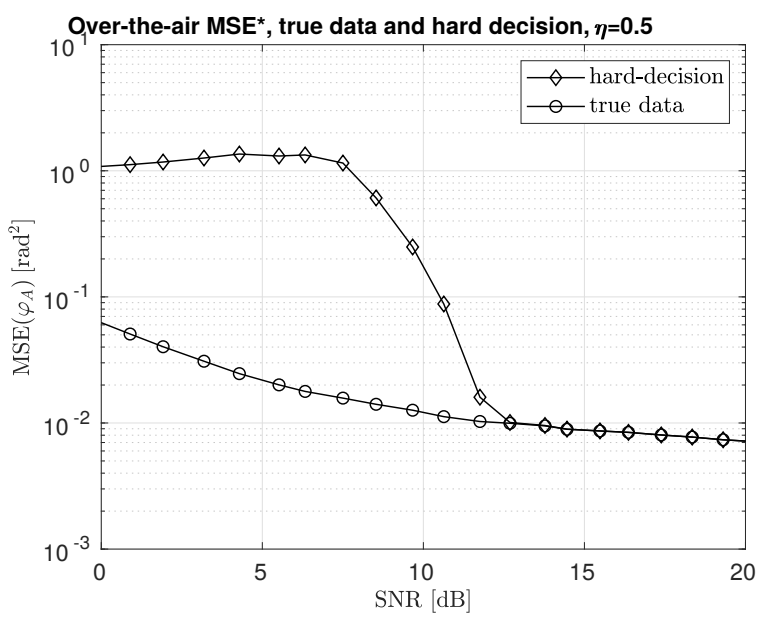

Fig. 11. Comparison of the MSE* performance of the over-theair experiment in the ture hierarchical data aided and hard decision scenario for $\eta=0.5$.

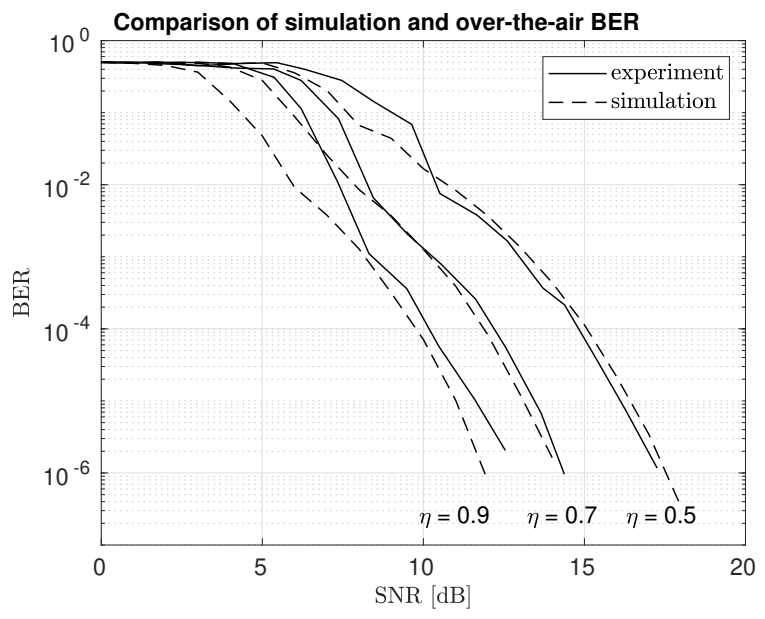

Fig. 12. Comparison of hard-decision BER evaluation for different values of $\eta \in\{0.5,0.7,0.9\}$ 


\section{Conclusion}

In the article we: 1) shortly extended the phase estimation limits from [7] by exploring the CRLB of constant phase estimator in an H-MAC channel. 2) The modification of the constant phase estimator for the variable phase was presented. 3) Performance of the variable phase estimator on software radios in terms of modified MSE; with and without the help of true H-data was shown.

\section{Acknowledgments}

Research was supported by the grant MSMT-LTC17042 of the Ministry of Education, Youth and Sports of the Czech Republic and by the grant SGS20/068/OHK3/1T/13 of Czech Technical University in Prague.

\section{Reference}

[1] SYKORA, J., BURR, A. Wireless Physical Layer Network Coding. Cambridge University Press, 2018. ISBN: 9781107096110

[2] ZHANG, S., LIEW, S. C., LAM, P. P. Hot topic: Physical-layer network coding. In Proceedings of the 12th Annual International Conference on Mobile Computing and Networking (MobiCom). New York (USA), 2006, p. 358-365. DOI: 10.1145/1161089.1161129

[3] KOIKE-AKINO, T., POPOVSKI, P., TAROKH, V. Optimized constellations for two-way wireless relaying with physical network coding. IEEE Journal on Selected Areas in Communications, 2009 vol. 27, no. 5, p. 773-787. DOI: 10.1109/JSAC.2009.090617

[4] NAZER, B., GASTPAR, M. Compute-and-forward: Harnessing interference through structured codes. IEEE Transactions on Information Theory, 2011, vol. 57, no. 10, p. 6463-6486. DOI: 10.1109/TIT.2011.2165816

[5] SYKORA, J., BURR, A. Layered design of hierarchical exclusive codebook and its capacity regions for HDF strategy in parametric wireless 2-WRC. IEEE Transactions on Vehicular Technology, 2011, vol. 60, no. 7, p. 3241-3252. DOI: 10.1109/TVT.2011.2160105
[6] SYKORA, J. Hierarchical data decision aided 2-source BPSK H-MAC channel phase estimator with feed-back gradient solver for WPNC networks. In Proceedings of the 14th International Conference on Wireless and Mobile Computing, Networking and Communications (WiMob). Limassol (Cyprus), 2018, p. 89-96. DOI: 10.1109/WiMOB.2018.8589117

[7] HRON, P., SYKORA, J. Performance analysis of hierarchical decision aided 2-source BPSK H-MAC CSE with feed-back gradient solver for WPNC networks. In Proceedings of the IEEE Microwave Theory and Techniques in Wireless Communications (MTTW). Riga (Latvia), 2019, p. 72-75. DOI: 10.1109/MTTW.2019.8897247

[8] YANG, Q., LIEW, S. C., LU, L., et al. Symbol misalignment estimation in asynchronous physical-layer network coding. IEEE Transactions on Vehicular Technology, 2017, vol. 66, no. 3, p. 2844-2852. DOI: 10.1109/TVT.2016.2578310

[9] DANG, X., LI, Q., YU, X. Symbol timing estimation for physicallayer network coding. IEEE Communications Letters, 2015, vol. 19, no. 5, p. 755-758. DOI: 10.1109/LCOMM.2015.2412931

\section{About the Authors...}

Petr HRON was born in 1994. His research is focused on the synchronization in wireless cloud networks. He received his master degree at the Faculty of Electrical Engineering, Czech Technical University in Prague in 2019.

Jozef LUKAC was born in 1995. He received his master degree at the Faculty of Electrical Engineering, Czech Technical University in Prague in 2019. His research interests include signal processing in wireless networks.

Jan SYKORA is associate professor at the Czech Technical University in Prague, Faculty of Electrical Engineering, Department of Radioelectronics. His research interest spans the whole area of the digital communication theory, including modulation, coding, synchronization, equalization and detection. 